\title{
Sensors and Micromachines-Key Components in Future Systems
}

\section{Ingemar Lundström}

Laboratory of Applied Physics

Linköping Institute of Technology

\begin{abstract}
The Institute of Electrical Engineering of Japan has recently started a Sensor and Micromachine Division and launched a new journal for the division. I have been asked to give some personal comments on this occasion especially related to the situation in Europe.
\end{abstract}

It is of large interest to the engineering community that the Institute has started a division on sensors and micromachines. Sensors, actuator and micromachine research, development and applications are found all over the world. This was manifested not least by the latest international conference on the subject, Transducers '95 Eurosensors IX held in Stockholm in the end of June 1995. The conference had over 1100 participants and about 540 scientific contributions. The various sessions at the conference were related to different aspects of sensors, actuators and micromachine research. This included general and theoretical aspects of sensors and actuators, micro electro-mechanical and micro-analytical systems, mechanical sensors, gas and ion sensors, biosensors, and novel materials. An evening session was devoted to the industrialization of sensors, where different aspects of the commercialization and marketing of new sensor technologies were described.

Sensors and actuators are thus already finding many industrial applications and will serve as key devices in many control-and measurement systems. Many of the proposed micromachines are imaginative with potential applications in several areas. The combination of (electrical) measurements, feed back and small mechanical devices will be very powerful. Sensors, actuators and micromachines are also finding use in many consumer products like cars, dishwashers, microwave ovens etc. Furthermore, several such devices are used by medical doctors for diagnosis and patient control and repair of damaged tissue. It is likely that we very soon will find "intelligent" microrobots and microtools combining physical and chemical sensors with micromachines and microactuators. New microanalytical diagnostic systems will be developed to make medical tests faster and more versatile.
Research on sensors and micromachines is truly interdisciplinary. It involves materials science, device physics and technology, mechanics and fluid dynamics, system- and control engineering, chemistry, biology medicine, and environmental science to mention a few of the areas. The development of sensors, actuators and micromachines needs therefore interdisciplinary collaboration. It is interesting to note that there exist already research institutes or university departments where such interdisciplinary research is performed. Interestingly such interdisciplinary research groups were formed very early in Europe. Furthermore, many research projects sponsored by the European Commission and present applications at the Commission aim at such interdisciplinary collaboration involving several European research institutes, universities and companies. It is likely that such collaborations will be extended in the future to involve not only European research groups. In areas related to e.g. environmental monitoring, sensors and actuators for automobiles and for medical uses the development would certainly benefit from international collaboration. It is worth noting that research on sensors, actuators and micromachines is rather strong in Europe and that some of the commercial development has been and is taking place in Europe. The interest in the field is further manifested by the early Eurosensors Conferences. The first special journal in the field was also published in Europe.

Thus there exist already special journals in the area of sensors, actuators and micromachines. A new journal would therefore seem to be unnecessary. I believe, however, that a knowledge of sensors, actuators and micromachines will be very important for almost all engineers in the future. Such devices will form the basis for new developments involving both electrical, mechanical, (bio)chemical and medical engineering. The start of the Sensor- and Micromachine Division with an accompanying new journal may be very important in this connection. 